\section{Morphological characteristics and clinical manifestations of orbital emphysema caused by isolated medial orbital wall fractures}

$\mathrm{H}$ Moon ${ }^{1}, \mathrm{Y} \mathrm{Kim}{ }^{2}, \mathrm{JM} \mathrm{Wi}{ }^{2}$ and $\mathrm{M} \mathrm{Chi}^{2}$
${ }^{1}$ Yeonsu Ever Bright Eye

Clinic, Incheon, Korea

${ }^{2}$ Department of Ophthalmology, Gachon University Gil Hospital, Incheon, Korea

Correspondence: M Chi, Department of Ophthalmology, Gachon University Gil Hospital, \#1198, Kuwol-dong, Namdong-ku, Incheon 405-760, Korea Tel: +82 32460 3364; Fax: +82324603358. E-mail: cmj@gilhospital.com

Received: 28 April 2015 Accepted in revised form: 8 December 2015 Published online: 22 January 2016

\begin{abstract}
Purpose To investigate the morphological characteristics and clinical manifestations of orbital emphysema in patients with isolated medial orbital wall fractures.

Methods This was a retrospective observational case series of $\mathbf{3 4 8}$ orbits of 348 patients with isolated medial orbital wall fractures. Medical charts were reviewed, and computed tomographic (CT) images were examined to determine the morphological characteristics of orbital emphysema.

Results Orbital emphysema was detected in 70 orbits $(20.1 \%)$. Large and communited type fracture was related with the presence of orbital emphysema $(P<0.05)$. Orbital air pockets were detected in medial or superior extraconal orbital segment in all cases with orbital emphysema. Swollen eyelid with crepitus $(\mathbf{9 0 . 0} \%)$ and supraduction limitation $(31.4 \%)$ were developed with orbital emphysema. All cases with supraduction limitation accompanied with superior extraconal orbital emphysema and superior rectus muscle deviation, and these eyes were fully recovered with conservative management without surgery. Conclusions Orbital emphysema can be a cause of ocular motility restriction following orbital wall fracture. If supraduction limitation is noted with isolated medial wall fracture and superior orbital emphysema with superior rectus muscle deviation is detected by CT scan, conservative management can be a good choice for spontaneous recovery delaying the surgery. Eye (2016) 30, 582-587; doi:10.1038/eye.2015.285; published online 22 January 2016
\end{abstract}

Introduction

Orbital emphysema is defined as the presence of air in orbit or periorbital tissues. The most common condition under which orbital emphysema develops is fractures in the paranasal sinuses and medial orbital wall along with valsalva maneuvers such as nose blowing or sneezing during the early posttraumatic periods. Orbital emphysema is often regarded as a self-limiting condition requiring no treatment and is not typically associated with any complications. ${ }^{1-7}$

Although some orbital emphysema cases with severe complications were reported, there has been no study that analyzed large case series of orbital emphysema. ${ }^{8-11}$ The present study investigated the morphological characteristics and clinical manifestations of orbital emphysema in patients with isolated medial orbital wall fractures. This study included orbital wall fractures with only medial wall, to simplify the effect of emphysema on the clinical manifestations of orbital wall fractures.

\section{Materials and methods}

Medical records of the patients with isolated medial orbital wall fractures who visited the emergency room of Gachon University Gil Hospital between January 2012 and December 2014 were retrospectively reviewed. The medical records were reviewed to obtain information about the patient age, gender, visual acuity, intraocular pressure, and signs and symptoms such as eyeball movement limitation, strabismus, and exophthalmos. 
All patients underwent computed tomography (CT) scans (axial, coronal, and sagittal orbital cuts 2-mm each) at emergency room. CT scans were reviewed to determine the type and size of medial wall fracture. The type of fracture was described as linear, hinged, or communited. The size of the fractured medial wall was categorized as a large, intermediate, or small fracture (Figure 1a). If the presence of orbital emphysema was checked, CT scans were further analyzed to evaluate the morphological characteristics of orbital emphysema. To determine the location of orbital air, the retrobulbar orbital space was divided into intraconal and extraconal areas and the extraconal area was further divided into medial, lateral, superior, and inferior segments
(Figure 1b). Especially, the rectus muscle or eyeball deviation by orbital emphysema was carefully examined. CT scans were also reviewed to detect the presence of subcutaneous air in the soft tissue of the upper and lower eyelids. Accompanying orbital injuries such as orbital or retrobulbar hemorrhage, extraocular muscle swelling or incarceration into the fracture site were also examined.

Patients were managed conservatively with antibiotic topical eyedrop, oral medications including antibiotic, anti-inflammatory and pain control drugs and cold compress for eyelid swelling, except surgically indicated cases.
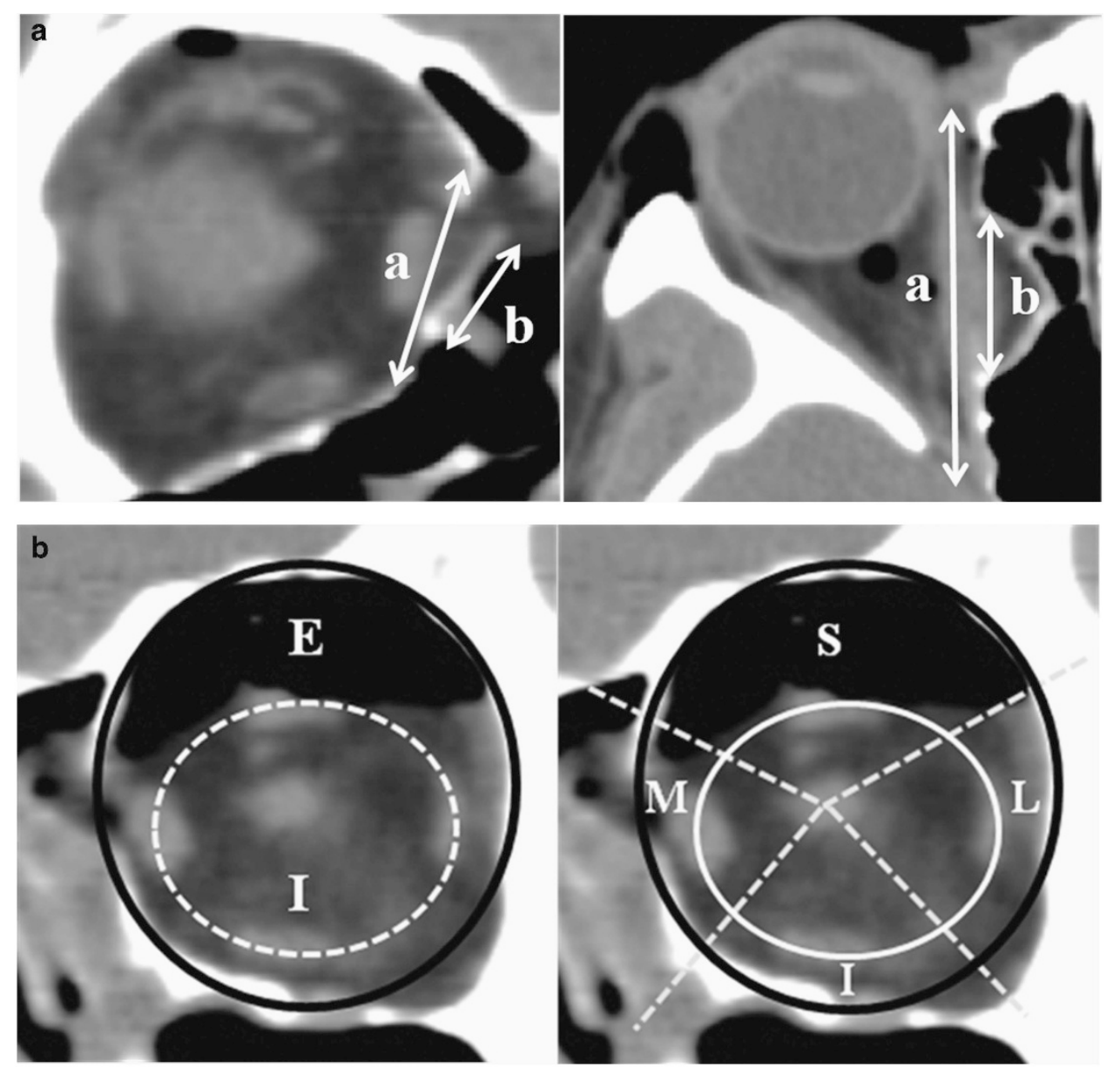

Figure 1 Determining the size of medial wall fracture (a) and sectorial classification of extraconal and intraconal orbital areas (b). (a) Determining the size of medial wall fracture. A coronal cut (left) and an axial cut (right) of CT scan. In each cut, 'a' represents the length of medial orbital wall and ' $b$ ' represents the length of fracture. The size of fracture was categorized as large, intermediate, or small according to the length of the fracture in each coronal and axial cut. If ' $b$ ' was longer than half of ' $a$ ' in both coronal and axial cuts, fracture size was determined as large. If ' $b$ ' was not longer than half of ' $a$ ' in both coronal and axial cuts, fracture size was determined as small. The others were regarded as intermediate size fracture. This case was classified as having intermediate fracture. (b) Sectorial classification of extraconal and intraconal orbital areas in a coronal cut of the soft tissue window. (Left) The intraconal (I) and extraconal (E) orbital areas of the retrobulbar space were divided by a virtual round line that connected the center of extraocular muscles (dotted line). (Right) The extraconal orbital space was divided into superior (S), medial (M), inferior (I), and lateral (L) segments according to virtual lines drawn from the optic nerve to the midline of the two adjacent rectus muscles in the coronal CT scan (dotted lines). 
Statistical analysis of all data was performed using Fisher's exact test. A $P$-value $<0.05$ was considered to be statistically significant.

\section{Results}

This study included 348 orbits of 348 patients, 275 men and 73 women, with isolated medial wall fractures. Orbital emphysema was detected in 70 orbits of 70 patients $(20.1 \%), 58$ men and 12 women, and the mean age was $35.4 \pm 14.5$ years. The presence of orbital emphysema was related with large size medial wall fracture $(P=0.001)$ and communited fracture $(P=0.040)$, and the presence of subcutaneous emphysema was related with the presence of orbital emphysema $(P<0.0001)$ (Table 1).

The morphological characteristics and clinical manifestations of the 70 orbits with orbital emphyhsema were further evaluated. The location of orbital emphysema was in extraconal orbital segments in all 70 orbits; 17 orbits $(24.3 \%)$ also had intraconal orbital air pockets. Among the 70 cases of extraconal orbital emphysema, orbital air was detected in the superior extraconal orbital segment in 49 (70.0\%), in the medial segment in $58(82.9 \%)$, in the inferior segment in $9(12.9 \%)$, and in the lateral segment in $4(5.7 \%)$ orbits. All eyes with orbital emphysema had the air pockets in medial or superior extraconal orbital segments. Especially, the superior rectus muscle or eyeball deviated downward due to the superior extraconal orbital emphysema was detected in 23 orbits (32.9\%).

Table 1 Relation between orbital emphysema, character of fracture and subcutaneous emphysema in isolated medial wall fracture

\begin{tabular}{|c|c|c|c|}
\hline & \multicolumn{2}{|c|}{ Orbital emphysema } & \multirow[t]{2}{*}{ P-value } \\
\hline & Absent & Present & \\
\hline Number of orbits & 278 & 70 & \\
\hline \multicolumn{4}{|l|}{ Size of fracture } \\
\hline small & $87(31.3 \%)$ & $7(10.0 \%)$ & 0.0003 \\
\hline intermediate & $65(23.4 \%)$ & $15(21.4 \%)$ & 0.729 \\
\hline large & $126(45.3 \%)$ & $48(68.6 \%)$ & 0.001 \\
\hline Size of fracture (trend) & & & 0.0002 \\
\hline \multicolumn{4}{|l|}{ Type of fracture } \\
\hline communited & $157(56.5 \%)$ & $49(70.0 \%)$ & 0.040 \\
\hline hinged & $92(33.1 \%)$ & $20(28.6 \%)$ & 0.469 \\
\hline linear & $29(10.4 \%)$ & $1(1.4 \%)$ & 0.016 \\
\hline Type of fracture (trend) & & & 0.0165 \\
\hline \multicolumn{4}{|l|}{ Subcutaneous empysema } \\
\hline abscent & $265(95.3 \%)$ & $7(10.0)$ & $<0.0001$ \\
\hline present & $13(4.7 \%)$ & $63(90.0 \%)$ & $<0.0001$ \\
\hline Subcutaneous empysema (trend) & & & $<0.0001$ \\
\hline
\end{tabular}

The most common sign caused by orbital emphysema was swollen eyelid with crepitus, which was detected in 63 orbits ( $90 \%$ in the 70 orbits with orbital emphysema), and subcutaneous emphysema was demonstrated by CT scans in these orbits.

The second common sign was limited eyeball movement, which was reported in 29 orbits; 22 orbits had supraduction limitation and 7 orbits had abduction or adduction limitation. We focused on the 22 orbits with supraduction limitation, because supraduction limitation is not common in isolated medial wall fracture. In the 22 orbits with supraduction limitation, all orbits had medial and superior extraconal orbital emphysema, and the superior rectus muscle deviated downward due to the compression caused by superior extraconal orbital emphysema. In these orbits, superior or inferior rectus muscle injury such as muscle swelling or intramuscular hemorrhage was not detected in CT scans. Because all study subjects had intact orbital floor, orbital emphysema could be one cause of supraduction limitation. All of these patients had blown their nose or sneezed immediately after trauma. These patients also had eyelid swelling with crepitus at palpation, and complained of diplopia when gazing upward. They were managed conservatively and fully recovered with no supraduction limitations, no eyelid swelling, no crepitus at palpation, and no diplopia within 7 days in all cases. A representative case is shown in Figure 2. The incidence of supraduction limitation was 31.4\% in the 70 orbits with orbital emphysema and $6.3 \%$ in the all 348 orbits with medial wall fractures.

Seven orbits with horizontal movement limitation were related with prolapse of the medial rectus muscle or surrounding soft tissue through the fractured medial orbital wall, and they were recovered after orbital wall reconstruction surgery.

Exophthalmometric measures showed enopthalmos in 16 orbits and exophthalmos from 0.5 to $1.5 \mathrm{~mm}$ in 7 orbits. Hypotropic eyeball deviation $<10$ prism diopters at primary gaze was observed in two orbits. The CT scan of these two orbits revealed orbital emphysema in the superior extraconal orbital space, which had compressed the superior rectus muscle and eyeball downward.

\section{Discussion}

In this study, we evaluated CT images to determine the morphological characteristics of orbital emphysema and its relationship to clinical manifestations. We discovered that the incidence of orbital emphysema was $20.1 \%$ with isolated medial wall fractures. Supraduction limitation was detected, and superior 

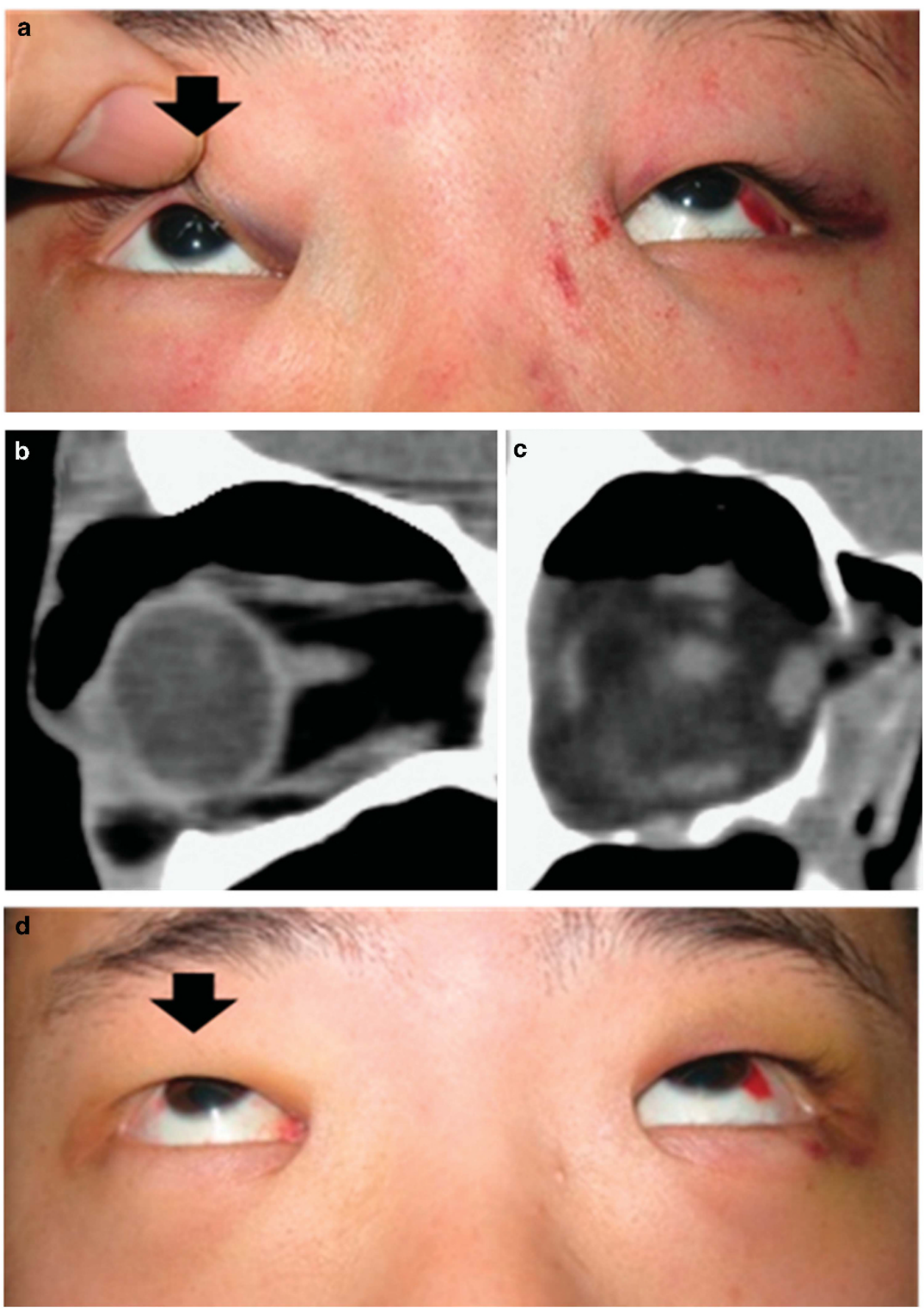

Figure 2 A case with supraduction limitation induced by orbital emphysema. A 14-year-old boy was hit in both eyes and blew his nose due to a nose-bleed immediately after the trauma. (a) On examination, both eyelids were swollen, crepitus was found in the right upper eyelid, and supraduction limitation was observed in the right eye (arrow). (b, c) A sagittal (b) and coronal (c) CT cuts showed a medial wall fracture, orbital emphysema in the superior extraconal segment that compressed the eyeball and superior rectus muscle downward, and subcutaneous emphysema in the upper eyelid of the right eye. There was no fracture or emphysema in the left eye. (d) The patient underwent conservative management, and supraduction limitation and crepitus of the eyelid disappeared 7 days after the trauma (arrow).

extraconal orbital emphysema and the superior rectus muscle deviated downward due to the orbital air was identified in these eyes. These patients fully recovered with no supraduction limitation, no eyelid swelling, no crepitus at palpation, and no diplopia within 7 days in all cases.

Air in the orbit can be detected by radiographic examination, most precisely by $\mathrm{CT}^{4}{ }^{4}$ Radiographic 
findings are helpful for understanding the mechanism underlying the development of orbital emphysema. In some radiographic studies, it has been speculated that nose blowing or sneezing during the early posttraumatic period induces increased intranasal air pressure. Air in the paranasal sinuses enters into the orbit through a pathway formed by disrupted sinus mucosa, fractured sinuses and orbital wall, injured periorbital tissues, and orbital septum. ${ }^{12-16}$ Disrupted orbital tissue, such as fat, acts as a one-way valve by falling back on this pathway and blocking the exit of air in the orbit. ${ }^{17}$ In the current study, we found that orbital emphysema was found in the medial or superior extraconal orbital space in all 70 cases we examined; 17 cases also had intraconal orbital air pockets. These findings may help elucidate a more detailed mechanism to explain the development and consequences of orbital emphysema than previous understandings. ${ }^{8}$ It may be that air from the paranasal sinus first enters into the medial extraconal orbital segments, and migrates to the superior extraconal orbital space by the force of gravity. If more forceful air pressure is generated, the air can infiltrate into superior, inferior, or lateral extraconal orbital segments, and extraconal orbital air can also enter into the intraconal space if the intermuscular septum is disrupted.

Indications for orbital wall reconstruction surgery include primary gaze diplopia, limited eyeball movement, enophthalmos more than $2 \mathrm{~mm}$, and large fracture. ${ }^{18}$ It is important to investigate the cause of eyeball movement limitation during the early posttraumatic period because early surgical management is required in cases with extraocular muscles or orbital soft tissue incarceration into the fracture site, and these findings can be easily detected by CT scans. However, some cases with eyeball movement limitation need no surgical management. Cases with extraocular muscle paralysis or swelling, orbital soft tissue edema, or orbital hemorrhage can be managed conservatively waiting for spontaneous recovery of the symptoms. ${ }^{18}$ The present study showed that orbital emphysema can be an another reversible cause of eyeball movement limitation and related diplopia. CT scan is a useful method to evaluate the characteristics of orbital emphysema and to reduce a potential misdiagnosis of restricted ocular motility following orbital fracture.

In summary, if supraduction limitation is noted with isolated medial wall fracture and superior orbital emphysema with superior rectus muscle deviation is detected by CT scan, conservative management can be a good choice for spontaneous recovery delaying the surgery regardless of muscle or soft tissue inflammation.

\section{Summary}

What was known before

- Orbital emphysema is often regarded as a self-limiting condition requiring no treatment.

- There has been no study that analyzed large case series of orbital emphysema.

What this study adds

- Orbital emphysema can be a cause of ocular motility restriction following orbital wall fracture.

- If supraduction limitation is noted with isolated medial wall fracture and superior orbital emphysema with superior rectus muscle deviation is detected by computed tomography scan, conservative management can be a good choice for spontaneous recovery delaying the surgery.

\section{Conflict of interest}

The authors declare no conflict of interest.

\section{References}

1 Lloyd GA. Orbital emphysema. Br J Radiol 1966; 39: 933-938.

2 Cruz AAV, Eichenberger GC. Epidemiology and management of orbital fractures. Curr Opin Ophthalmol 2004; 15: 416-421.

3 Başterzi Y, Sari A, Yavuzer R, Atabay K. Periorbital subcutaneous emphysema: an unusual clinical presentation of medial orbital wall fracture. Plast Reconstr Surg 2001; 108: 2156-2157.

4 Lee HJ, Jilani M, Frohman L, Baker S. CT of orbital trauma. Emerg Radiol 2004; 10: 168-172.

5 Muhammad JK, Simpson MT. Orbital emphysema and the medial orbital wall: a review of the literature with particular reference to that associated with indirect trauma and possible blindness. J Craniomaxillofac Surg 1996; 24: 245-250.

6 Segrest DR, Dortzbach RK. Medial orbital wall fractures: complications and management. Ophthal Plast Reconstr Surg 1989; 5: 75-80.

7 Van Issum C, Courvoisier DS, Scolozzi P. Posttraumatic orbital emphysema: incidence, topographic classification and possible pathophysiologic mechanisms. A retrospective study of 137 patients. Oral Surg Oral Med Oral Pathol Oral Radiol 2013; 115: 737-742.

8 Key SJ, Ryba F, Holmes S, Manisali M. Orbital emphysemathe need for surgical intervention. J Craniomaxillofac Surg 2008; 36: 473-476.

9 Ahnood D, Toft PB. Recurrent orbital compartment syndrome caused by a blow-out fracture and accumulation of air; management by orbital punctures. Acta Ophthalmologica 2012; 90: 199-200.

10 Tomasetti R, Jacobsen C, Gander T, Zemann W. Emergency decompression of tension retrobulbar emphysema secondary to orbital floor fracture. J Surg Case Rep 2013; e-pub ahead of print 21 March 2013; doi:10.1093/jscr/rjt011.

11 Zimmer-Galler IE, Bartley GB. Orbital emphysema: case reports and review of the literature. Mayo Clin Proc 1994; 69: 115-121. 
12 Ord RA, May ML, Duncan JG, Moos KF. Computerized tomography and B-scan ultrasonography in the diagnosis of fractures of the medial orbital wall. Plast Reconstr Surg 1981; 67: 281-288.

13 Evans GRD, Clark N, Manson PN. Identification and management of minimally displaced nasoethmoidal orbital fractures. Ann Plast Surg 1995; 35: 469-473.

14 Shinohara H, Shirota Y, Fujita K. Implication of differences in the incidence of orbital emphysema in ethmoidal and maxillary sinus fractures. Ann Plast Surg 2004; 53: $565-569$.
15 Burm JS, Chung CH, Oh SJ. Pure orbital blowout fracture: new concept and importance of medial orbital blowout fracture. Plast Reconstr Surg 1999; 103: 1839-1849.

16 Castelnuovo P, Mauri S, Bignami M. Spontaneous compressive orbital emphysema of rhinogenic origin. Eur Arch Otorhinolaryngol 2000; 257: 533-636.

17 Hunts JH, Patrinely JR, Holds JB, Anderson RL. Orbital emphysema. Staging and acute management. Ophthalmology 1994; 101: 960-966.

18 Cole P, Boyd V, Banerji S, Hollier LH Jr. Comprehensive management of orbital fractures. Plast Reconstr Surg 2007; 120: 57S-63S. 